\title{
Hinode observations of transverse waves with flows in coronal loops
}

\author{
L. Ofman and T. J. Wang \\ Dept. of Physics, The Catholic University of America and NASA Goddard Space Flight Center, Code 671, Greenbelt, MD 20771, \\ USA \\ e-mail: ofman@waves.gsfc . nasa.gov
}

Received 28 December 2007 / Accepted 12 March 2008

\section{ABSTRACT}

\begin{abstract}
Aims. We report the first evidence for transverse waves in coronal multithreaded loops with cool plasma ejected from the chromosphere flowing along the threads. These observations are good candidates for coronal seismology.

Methods. We analyzed observations made with Solar Optical Telescope (SOT) on board the Hinode satellite in the Ca II H line filter. Results. The oscillations are visible for about 3 periods, with a period lasting about 2 min, with weak damping. We see the oscillations in thin threads $\left(\sim 0.5^{\prime \prime}\right)$ of cool plasma flowing in the coronal loops with speeds in the range $74-123 \mathrm{~km} \mathrm{~s}^{-1}$.

Conclusions. Observations indicate that the waves exhibit different properties in the various threads. In some threads, the waves are nearly standing fundamental kink modes with a phase speed of about $1250 \mathrm{~km} \mathrm{~s}^{-1}$, whereas the dynamics of other threads is consistent with propagating fast magnetosonic waves. Based on the observed wave and loop properties and the assumed active region loop density in the range $(1-5) \times 10^{9} \mathrm{~cm}^{-3}$, the estimated energy flux is sufficient to heat the loops to coronal temperatures, and the average magnetic field in the threads is estimated as $20 \pm 7 \mathrm{G}$.
\end{abstract}

Key words. Sun: flares - Sun: corona - Sun: magnetic fields - Sun: oscillations - waves

\section{Introduction}

The Solar Optical Telescope (SOT) on board the Hinode (SolarB) satellite (The Solar-B Team 2005) consists of a 50-cm aperture, diffraction-limited Gregorian telescope with spatial resolution of $0.2-0.3^{\prime \prime}$ and cadence of $1 \sim 10 \mathrm{~s}$ (Ichimoto \& Sot Team 2005). In the present letter, we analyze Hinode/SOT data of coronal loop oscillations obtained on 12 Jan. 2007, which was visible in the $\mathrm{Ca}$ II $\mathrm{H}$ emission line filter due to the flow of cool chromospheric material in the coronal loop. We utilize the data for coronal seismology. The upflow of chromospheric material and the oscillations are related to a flare that occurs near the footpoints of the observed loop, seen by the Solar and Heliospheric Observatory (SOHO) Extreme ultraviolet Imaging Telescope (EIT) and is evident in Geostationary Operations Environmental Satellite (GOES) X-ray data.

The method of coronal seismology was first discussed by Uchida (1970) and Roberts et al. (1984) in the context of coronal loops. Similar to helioseismology, the method is based on observations of waves in coronal structures, and on the use of theoretical knowledge and computational modeling of the wave properties to extract the physical parameters of the coronal structures, such as the poorly known magnetic field. However, in contrast to helioseismology the method is complicated by the coupling of the three magnetohydrodynamic (MHD) wave modes in nonhomogeneous plasma, as well as by nonlinearity. The method requires high spatio-temporal resolution observations of the corona to resolve the wave motions that only became routinely available with the launch of SOHO spacecraft, and the Transition Region and Coronal Explorer (TRACE) satellite. The method of coronal seismology was applied to TRACE and SOHO data (e.g., Nakariakov et al. 1999; Nakariakov \& Ofman 2001; Ofman 2002; De Moortel et al. 2002; Ofman \& Wang 2002;
Ofman \& Thompson 2002; Verwichte et al. 2004; Wang \& Solanki 2004; Aschwanden 2005), with recent advances (e.g., Ofman 2007; Wang et al. 2007; Van Doorsselaere et al. 2007; Ballai 2007; Arregui et al. 2007).

Recently, Alfvén waves were observed in the transition region (De Pontieu et al. 2007) and in X-ray jets (Cirtain et al. 2007), transverse waves in solar prominence loops were observed by Okamoto et al. (2007) with Hinode SOT. Lowamplitude Alfvén waves with a directly-detected energy flux that is insufficient to heat the corona were detected throughout the corona (Tomczyk et al. 2007). In this letter, we report the first Hinode observation of weakly-damped coronal loop oscillation in the presence of background flow (Sect. 2), and use the observed parameters of the waves with assumed typical active region loop density to determine the average magnetic field in the loop, and the wave energy flux. The estimated wave energy flux is consistent with energy flux required to heat the loop to coronal temperatures. The discussion and summary of the results are in Sect. 3.

\section{Observations}

\subsection{Determination of loop parameters}

A filament eruption was detected by Hinode/SOT in the Ca II H emission line filter in the solar west limb on 12 Jan. 2007. It was associated with a GOES C1.5-class flare and a coronal mass ejection (CME). The flare started at 01:18 UT and peaked at 01:38 UT in soft X-rays and the CME was first seen by Large Angle and Spectrometric Coronagraph (LASCO) on SOHO at 01:54 UT. The Ca II H line (3968 $⿱$ A) images obtained with very high spatial $\left(\sim 0.2^{\prime \prime}\right)$ and temporal $(8 \mathrm{~s})$ resolutions, which allowed us to study the fine structures and the dynamics 


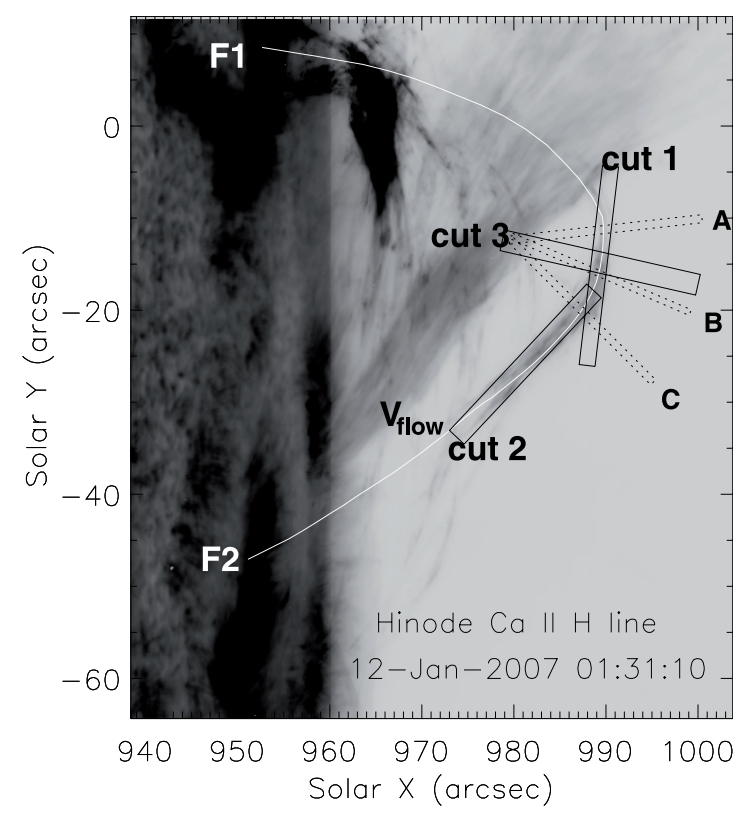

Fig. 1. Chromospheric material at 10000-20000 K in coronal loops observed by Hinode SOT with Ca II H emission line filter (3968.5 \pm $3 \AA$ ). The material flows along the loops, which exhibit periodic oscillations (the movie of this event is available online). The oscillating loop studied is outlined with a white curve, which is obtained by tracing flows observed at 01:28:15, 01:31:10, and 01:34:40. The plasmas were ejected from the region F1 and moved along the loop to the other foot F2. Cuts 1 and 2 are used to measure the flow speeds (see Fig. 2), cut 3 is used to measure the transverse oscillations of the loop (see Fig. 3), and cuts A-C (dotted narrow boxes) are used to detect the wave propagation (see Fig. 4). This image and subsequent images are "negative" images, and the darkest features correspond to brightest features in SOT.

of coronal loops and the chromospheric material in the corona. SOHO/EIT $195 \AA$ observations of this region at times close to the flare, and much lower resolution of $3^{\prime \prime}$ show that coronal loops are present there. Figure 1 shows that the chromospheric material at a temperature of 10000-20000 K, which was ejected out from the region marked as F1 during the filament eruption, moved along the magnetic loops and then fell down to the region marked as F2. The cool material appears to flow along thin threads of about $0.4-0.6^{\prime \prime}$ inside the loops at speeds in the range of $74-123 \mathrm{~km}^{-1} \mathrm{~s}$ (see below). The thin-threaded coronal loop structure is consistent with past models of coronal loops (e.g., Aschwanden et al. 2000; Ofman \& Aschwanden 2002). Coronal magnetic structures can be identified by tracing the flowing cool plasma in the consecutively observed images. We found a coronal loop outlined with a white curve to oscillate transversely to its axis, during which the cool plasma was flowing along the loop. Using the Hinode/SOT images we measured the projected length of the oscillating loop to be about $71 \mathrm{Mm}$, the height about $27 \mathrm{Mm}$, and the feet separation about $40 \mathrm{Mm}$. We estimate the uncertainty in the loop length to be about $10-20 \%$ due to projection, and line of sight effects. However, this uncertainty is negligible in our analysis below compared to uncertainty introduced by the estimated range of densities.

\subsection{Flow velocity measurements in the loop}

In order to measure the flow speed in the oscillating loop, we took two cuts along the loop (marked 1 and 2 in Fig. 1) and

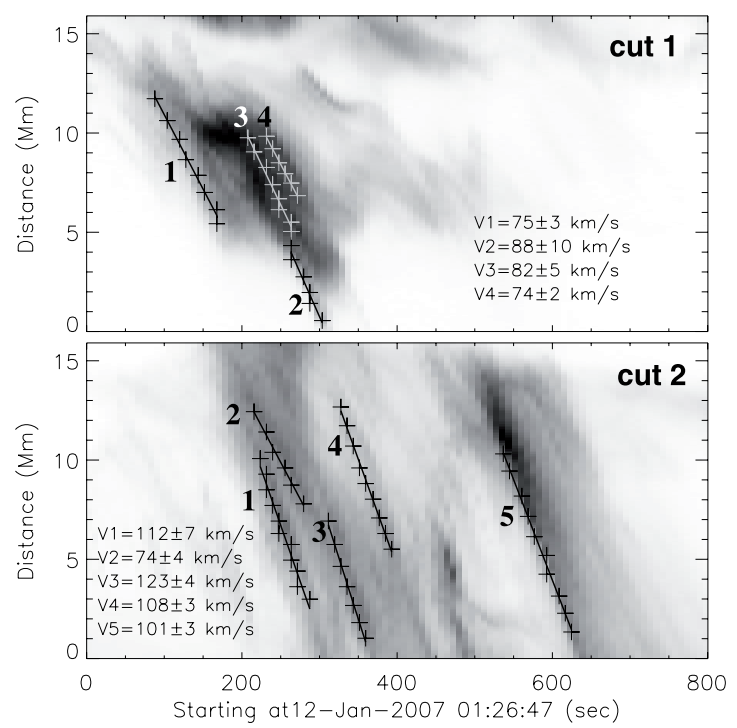

Fig. 2. Evolution of flow emissions along the oscillating loop at cuts 1 and 2 (see Fig. 1). The flow speeds are measured by tracking the motion of some brightening features seen as dark stripes in the negative image. The positions of prominent moving features are marked and fitted linearly, yielding the apparent flow speeds which are labeled on the map. On the vertical axis, 0 corresponds to the lower end of the cut.

plotted the time series of their emission intensity, averaged along the narrow side of the cut in Fig. 2. The blobs of cool plasma moving downward along the loop threads are shown as the dark stripes slanting to the right in the negative image. Four dark stripes in the time-distance map for cut 1 and five dark stripes in the map for cut 2 are marked. The flow speed for each moving plasma blob is measured by a linear fit. The measured values are labeled on the maps. For cut 1 the flow speed is on average $V_{\mathrm{f}}=80 \pm 7 \mathrm{~km} \mathrm{~s}^{-1}$ and for cut 2 the mean flow speed is $V_{\mathrm{f}}=104 \pm 18 \mathrm{~km} \mathrm{~s}^{-1}$. Note, that the flow of the moving blob 2 (V2) is considerably smaller than the average flow in cut 2 . At least part of this differences may be explained by the projection effect due to the angle formed by this thread with the average flow direction. The measurements indicate that the plasma flow is accelerating when it falls down from the loop top, consistent with the effect of solar gravitational acceleration. However, the change in velocity due to gravity is small compared to the flow velocity in this case, and there could be other effects that affect the flow speed (for example loop divergence, or change in density or temperature of the material).

\subsection{Evidence for waves in the loop}

From the time sequence of the images it is evident that the transverse oscillations are most pronounced in the top part of the loop. Figure 3 shows time series of emission intensity along a cut across the loop top (marked 3 in Fig. 1). To enhance the small scale features, the image is processed with a 3-by-3 high-pass (sharpening) filter. The oscillations are visible for about three periods and show a weak decay. We measure the physical parameters of the displacement oscillations by fitting the marked positions with a damped sine function. The oscillating loop is composed of several fine threads that merge and separate during the oscillation, leading to uncertainty in identifying the exact number of distinct threads in the loop. Since the observations are near the solar limb and the threads are seen to be displaced in the vertical direction in the plane of the sky, it is likely that 


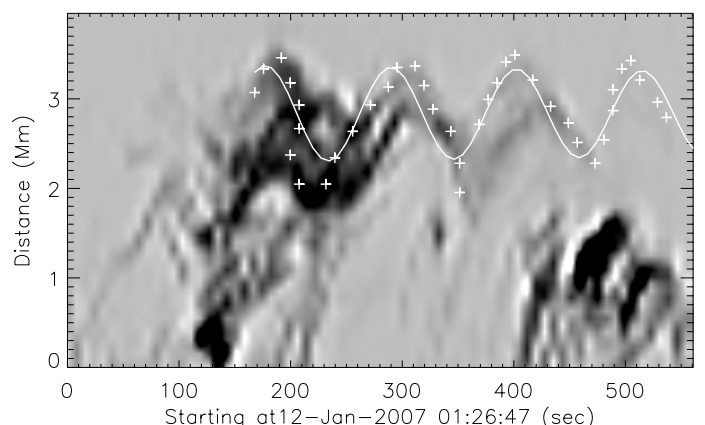

Fig. 3. Time series of the plasma emission in the Ca II H filter along Cut 3 across the loop top (see Fig. 1). For weak oscillatory features to be seen clearly, the image is processed by a sharpening filter. The positions of the oscillating loop are marked with "+" and best fitted with a damped sine function (white curve). The oscillations have a period of $113 \mathrm{~s}$ with weak decay.

the oscillations contain a vertical component, similar to the vertical oscillations recently observed by TRACE (Wang \& Solanki 2004).

The multithreaded structure of the loop, together with time variability of the flow and line of sight effects, introduce considerable uncertainty in the determination of distinct threads. Due to this uncertainty, in each case, we selected different positions in the loop, and averaged the resulting period over these observations. We find that the oscillation period $(P)$ has a mean value of $113 \pm 2 \mathrm{~s}$, the maximum displacement amplitude $\left(A_{\mathrm{m}}\right)$ is $0.67 \pm$ $0.12 \mathrm{Mm}$. The maximum velocity amplitude is estimated to be $37 \pm 7 \mathrm{~km} \mathrm{~s}^{-1}$ by $V_{\mathrm{m}}=2 \pi A_{\mathrm{m}} / P$. Also, the amplitude of the oscillations appears to peak near the top of the loop, suggesting that this is a fundamental mode. The average damping time of the oscillations $\left(\tau_{\mathrm{d}}\right)$ is $979 \pm 1060 \mathrm{~s}$. However, in one measurement, the damping time (see Fig. 3) has a very large uncertainty, with a value $\tau_{\mathrm{d}}=3075 \pm 3198 \mathrm{~s}$, due to negligible damping of the oscillations, and because only 3 full periods are seen. If we exclude this case, the decay time is on average $560 \pm 297 \mathrm{~s}$.

To examine whether the oscillations are propagating, we compare the phase relationship between three time series of intensities taken at cuts A-C shown in Fig. 1. Figure 4 shows that the erupted cool plasma first reached cut A and then cut B, followed by cut $\mathrm{C}$. We identify a thread of cool material that appears in both cuts, and find no significant phase displacement between the time sequences. The locations of peaks and valleys of threads exhibiting standing oscillations are shown with "S", followed by a number that identifies them in the three panels. Due to the large phase speed of the fast magnetosonic waves and the temporal resolution of SOT, the propagating waves can not be determined unambiguously in this figure. Observational line of sight effects, and temporal variability of the cool material flow, do not allow a unique determination of more than a few threads. If we assume the time spent for a propagating wave traveling from $\mathrm{A}$ to $\mathrm{C}$ is within measurement uncertainties of 1 frame, we can estimate its phase speed at least $\sim 900 \mathrm{~km} \mathrm{~s}^{-1}$, where the arc length of the loop between cuts A and C is $\sim 7000 \mathrm{~km}$. Thus, our results are consistent with either a propagating fast magnetosonic wave in an inhomogeneous medium with the speed above $900 \mathrm{~km} \mathrm{~s}^{-1}$ in some threads, or with a transverse kink mode wave (i.e., the oscillations are in phase at all cuts) in other threads. Since some threads do not appear to move in phase in the 3 cuts discussed above, the wave could be a propagating wave in those threads. Because the oscillations are mostly

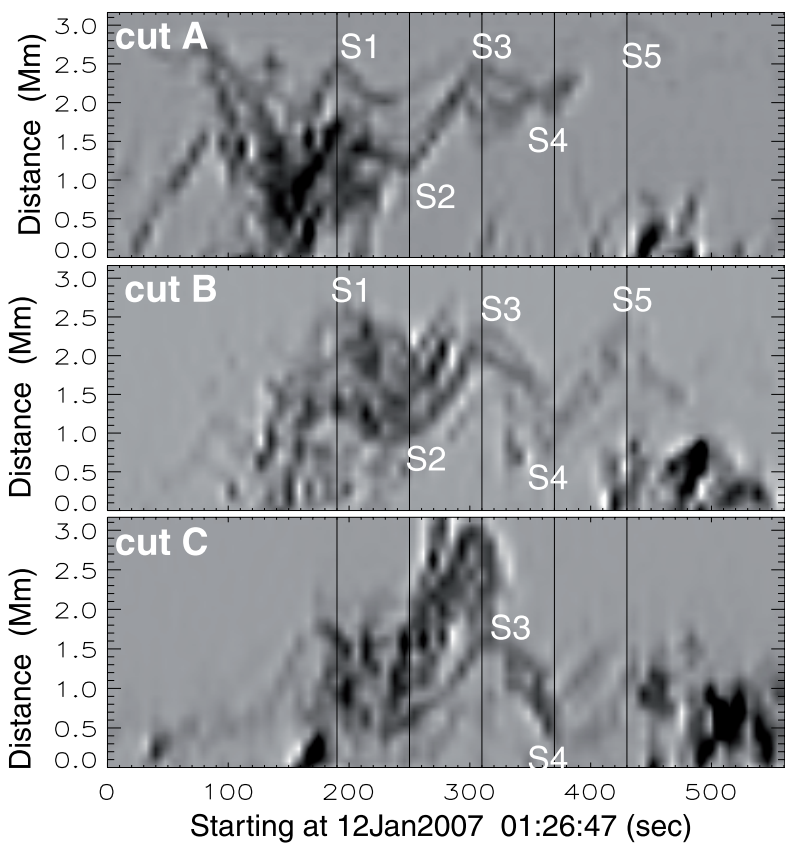

Fig. 4. Time series of the plasma emission along cuts A-C across the loop top (see in Fig. 1). Five vertical lines across the panels mark the times when the displacement reaches the maximum or minimum in order to compare their phase relationships at the 3 cuts. On the vertical axis, 0 corresponds to the end of the cut closer to the limb. The locations of peaks and vales of threads exhibiting standing oscillations are marked with "S", followed by a number.

prominent near the loop top, the wave is most likely the fundamental mode in the threads that oscillate in phase.

\subsection{Determination of magnetic field and energy}

If we assume that the oscillating loops are high density compared to the surrounding medium, and the oscillation is a fundamental kink mode in several threads, where the kink speed is $C_{\mathrm{k}}=2^{1 / 2} V_{\mathrm{A}}=2 L / P=1254 \mathrm{~km} \mathrm{~s}^{-1}$, and the Alfvén speed $V_{\mathrm{A}}=887 \mathrm{~km} \mathrm{~s}^{-1}$. Using the value of $V_{\mathrm{A}}$ to determine the average magnetic field in the loop, we get $B=(2 L / P) \sqrt{2 \pi n m_{\mathrm{p}}}$ with the observed values of $L$ and $P$, and we find that the magnetic field is in the range 13-27 G given that the average number density in the loop is in the range $n=(1-5) \times 10^{9} \mathrm{~cm}^{-3}$. The present observations do not allow us to determine the exact density of the loops. However, the values we use are in line with other observations of active region loops (e.g., Nakariakov \& Ofman 2001; Aschwanden 2005).

Based on the above determination of $V_{\mathrm{A}}$ (which is the lower bound for $C_{\mathrm{k}}$ ) and estimate of $n$, we can estimate the energy flux carried by the transverse waves. With the Wentzel-KramersBrillouin (WKB) approximation, the energy flux is given by $E_{\mathrm{W}}=\frac{1}{2} \rho(\delta V)^{2}\left(V_{\mathrm{A}} \pm\left|V_{\mathrm{f}}\right|\right)$, where $\delta V$ is the wave amplitude, and $\left|V_{\mathrm{f}}\right|$ is the magnitude of the flow along the loop. The sign is determined by the direction of the flow compared to the direction of the wave propagation. Substituting the observed values, we get $E_{\mathrm{w}}=7 \times 10^{5}-5 \times 10^{6} \mathrm{erg} \mathrm{cm}^{-2} \mathrm{~s}^{-1}$ with the above range of average number density in the loop. This energy flux can balance typical radiative and conductive losses (Withbroe \& Noyes 1977; Aschwanden 2005) of an active region coronal loop, and heat it to coronal temperature.

The required energy flux to heat a coronal loop to a particular coronal temperature on the order of $\sim 10^{6} \mathrm{~K}$ increases 
quadratically with density (e.g., Aschwanden 2005). However, since the Alfvén speed and the wave amplitude are determined from the observations independent of the density, the estimate of the wave energy flux depends linearly on density. Given that the estimated energy flux is sufficient to heat the loop with the assumed density range of $(1-5) \times 10^{9} \mathrm{~cm}^{-3}$, if the actual loop density is an order of magnitude lower than this range, the corresponding estimated energy flux would be an order of magnitude higher than needed to heat the loop to coronal temperature. Conversely, if the actual loop density is an order of magnitude larger than the assumed range, then the corresponding energy flux would be an order of magnitude lower than needed to heat the loop.

\section{Discussion and conclusions}

We report the first Hinode/SOT observation of coronal loop oscillation with background flow of chromospheric material. The cool chromospheric material traces the loops in thin threads and enables us to observe the fine structure and the oscillations in $\mathrm{Ca}$ II $\mathrm{H}$ line. The generation of the flows is related to the impulsive event (flare) that occurs in the vicinity of the observed loop footpoints, but the excitation mechanism of the waves is not evident because we did not observe the onset phase of the fast magnetosonic waves. We saw the flare in SOHO/EIT, and SXI/GOES-12 X-ray data obtained on 12 Jan. 2007. Transverse oscillations excited by flares and filament eruptions were seen previously with TRACE (e.g., Aschwanden 2005). However, there was no direct evidence of plasma flows in thin threads in the oscillating loops prior to our report in this letter.

We find that the oscillations are transverse to the loop axis, and that the phase speed of the oscillations agrees with the kink or fast magnetosonic speed in these loops. Since the corona is highly structured and low-beta; i.e., the ratio of thermal pressure to magnetic pressure is small $(\ll 1)$, an arbitrary motion of magnetic field will likely affect the density of the plasma. Therefore, in agreement with observations that show spatial and temporal density variations in the threads we interpret the waves in terms of fast magnetosonic waves. The near-limb loop oscillations likely contain a vertical component, similar to the vertical oscillations recently observed by TRACE (Wang \& Solanki 2004), and modeled numerically (Selwa et al. 2005; Gruszecki et al. 2006) without parallel flow. In low beta coronal plasma, the sound speed is on the order of $100 \mathrm{~km} \mathrm{~s}^{-1}$, an order of magnitude lower than $V_{\mathrm{A}}$. Therefore, we conclude that the oscillations are fast magnetosonic or kink oscillation with weak damping. The effect of the background flow is to modify the energy flux, and affect the conditions for the formation of a standing wave in the loop. In one loop thread the damping appears to be negligible, and the wave appears to be fully trapped in the loop. However, since the oscillations were observed only for three periods the damping stage of the oscillations was not observed. The weak damping, or even growth of oscillation amplitude for few periods followed by damping at later times was seen in TRACE loops (Aschwanden et al. 2002). In the other threads the damping time is about 5 times longer than the period, similar with some oscillating loops observed by TRACE (Aschwanden et al. 2002).

There are three main classes of wave damping mechanism that are currently debated in the literature (e.g., Roberts 2000; Ruderman \& Roberts 2002; Ofman 2002; Ofman \& Aschwanden 2002; Goossens et al. 2002; Van Doorsselaere et al. 2004; Aschwanden 2005): leakage, resonant absorption or mode conversion, and dissipation (i.e., conversion of the wave energy to heat). The present observation shows that the oscillations occur in thin threads inside the loop with slightly different properties, which are consistent with the conditions required by the phase mixing in multithreaded loops and enhanced resistivity as suggested by the Ofman \& Aschwanden (2002) analysis. The initial weak damping, or even growth of the wave amplitude followed by strong damping is expected in some cases with this mechanism determined by the interplay between the scale and geometry of the inhomogeneity, the density contrast, the magnetic field, and the effective damping coefficient (e.g., Smith et al. 2007). All of these quantities can vary between the threads.

Since our observations indicate that the fast wave propagates at a speed of $>1000 \mathrm{~km} \mathrm{~s}^{-1}$, only about half the period is required for the wave to traverse the length of the loop. The observations show that the threads are present for more then three periods more than sufficient time to establish kink oscillations. We use the properties of the kink mode oscillation in several threads in the loop to determine the average magnetic field in the range of 13-27 $\mathrm{G}$ (depending on the assumed value of average density in the $\left.(1-5) \times 10^{9} \mathrm{~cm}^{-3}\right)$. We also find that the energy flux contained in the waves can supply sufficient energy to heat the loops with this density to coronal temperatures. The main limitation of the above analysis is the use of an estimated active region loop density, since the actual loop density could not be determined in the present observation.

Acknowledgements. This work was supported by NASA grant NNG06GI55G, NRL contract N00173-06-1-G033, and by Sun-Earth Connection Theory Program.

\section{References}

Arregui, I., Andries, J., van Doorsselaere, T., Goossens, M., \& Poedts, S. 2007, A\&A, 463, 333

Aschwanden, M. J. 2005, Physics of the Solar Corona. An Introduction with Problems and Solutions, 2nd edn. (Springer-Praxis)

Aschwanden, M. J., Nightingale, R. W., \& Alexander, D. 2000, ApJ, 541, 1059

Aschwanden, M. J., De Pontieu, B., Schrijver, C. J., \& Title, A. M. 2002, Sol. Phys., 206, 99

Ballai, I. 2007, Sol. Phys., 72

Cirtain, J. W., Golub, L., Lundquist, L., et al. 2007, Science, 318, 1580

De Moortel, I., Hood, A. W., \& Ireland, J. 2002, A\&A, 381, 311

De Pontieu, B., McIntosh, S. W., Carlsson, M., et al. 2007, Science, 318, 1574

Goossens, M., Andries, J., \& Aschwanden, M. J. 2002, A\&A, 394, L39

Gruszecki, M., Murawski, K., Selwa, M., \& Ofman, L. 2006, A\&A, 460, 887

Ichimoto, K., \& Sot Team 2005, in Chromospheric and Coronal Magnetic Fields, ed. D. E. Innes, A. Lagg, \& S. A. Solanki, ESA Special Publication, 596

Nakariakov, V. M., \& Ofman, L. 2001, A\&A, 372, L53

Nakariakov, V. M., Ofman, L., DeLuca, E., Roberts, B., \& Davila, J. M. 1999, Science, 285, 862

Ofman, L. 2002, ApJ, 568, L135

Ofman, L. 2007, ApJ, 655, 1134

Ofman, L., \& Aschwanden, M. J. 2002, ApJ, 576, L153

Ofman, L., \& Thompson, B. J. 2002, ApJ, 574, 440

Ofman, L., \& Wang, T. J. 2002, ApJ, 580, L85

Okamoto, T. J., Tsuneta, S., Berger, T. E., et al. 2007, Science, 318, 1577

Roberts, B. 2000, Sol. Phys., 193, 139

Roberts, B., Edwin, P. M., \& Benz, A. O. 1984, ApJ, 279, 857

Ruderman, M. S., \& Roberts, B. 2002, ApJ, 577, 475

Selwa, M., Murawski, K., Solanki, S. K., Wang, T. J., \& Tóth, G. 2005, A\&A, 440, 385

Smith, P. D., Tsiklauri, D., \& Ruderman, M. S. 2007, A\&A, 475, 1111

The Solar-B Team 2005, Adv. Space Res., 36, 1478

Tomczyk, S., McIntosh, S. W., Keil, S. L., et al. 2007, Science, 317, 1192

Uchida, Y. 1970, PASJ, 22, 341

Van Doorsselaere, T., Andries, J., Poedts, S., \& Goossens, M. 2004, ApJ, 606, 1223

Van Doorsselaere, T., Nakariakov, V. M., \& Verwichte, E. 2007, A\&A, 473, 959 Verwichte, E., Nakariakov, V. M., Ofman, L., \& Deluca, E. E. 2004, Sol. Phys., 223, 77

Wang, T. J., \& Solanki, S. K. 2004, A\&A, 421, L33

Wang, T. J., Innes, D. E., \& Qiu, J. 2007, ApJ, 656, 598

Withbroe, G. L., \& Noyes, R. W. 1977, ARA\&A, 15, 363 